\title{
Monika Frommel \\ Feministische Rechtskritik und Rechtssoziologie - Rekonstruktion eines disziplinären Mißverständnisses
}

\author{
1. Das Bremer Projekt "Durchsetzungschancen gleichen Rechts für \\ Frauen: 1980-1990
}

1984 erschien im Schwerpunktheft der Zcitschrift für Rechtssoziologie »Frauen und Rechr «' ein Bericht von Ute Gerhard über ein gemeinsam mit Rüdiger Lautmann durchgeführtes Projekt. Folgt man dem Vorwort: "Dic hcrkömmliche Jurisprudenz darf pikanterweise ohne den geringsten Abstrich als eine Domäne maskuliner Kultur bezeichnet werden; sie befand sich auch immer in männlichen Händen - die rechtswissenschaft]ichen Lchrstühle beispielweise zu 99 Prozent " (Lautmann), dann wollten die Beteiligten die Gründe herausfinden, wieso die herkömmliche Jurisprudenz insofern der Theologie, Medizin, Philosophie und den Naturwissenschaften vergleichbar - nach wic vor so extrem männliche Orienticrungen vermittelt, obgleich sich, was dic juristischen Berufe außerhalb der Universitäten betrifft, so etwas wie eine "Feminisierung der juristischen Profession " abzeichne ${ }^{2}$. Wer nach den viclversprechenden Ankündigungen des Jahres 1984 dic beiden 1990 crschicnenen, aber getrennt veröffentlichten Ergebnisse der ursprünglich gemeinsamen Projektarbeit liest ${ }^{3}$, wundert sich, wieso beide den 1984 gesponnenen Faden nicht wieder aufnehmen, sondern stattdessen - teilweise unter Verwendung derselben Materialien - über die damals diskutierten Themen schreiben, ohne cxplizir und an sichrbarer Stelle aufeinander einzugehent.

Die kontroversen Ausgangspunkte zwischen Ute Gerhard und Rüdiger Lautmann bezichen sich im wcsentlichen auf das Postulat der Gleichbeit der Geschlechter und spitzen sich zu bei der Gretchenfrage nach den strategischen Kompetenzen von Fraucn im Umgang mit Recht. Läßrt sich die von beiden geteilte Beobachtung einer »skeptischen " (Gerhard) Haltung vieler Frauen gegenüber juristischen Instanzen um- und abwerten zu einem "negativem Rechtsbewußtsein (so Lautmann) oder zcigt umgekehrt die Skcpsis an, daß Frauen "Unrechtserfahrungen" gemacht haben und deshalb vorsichtiger geworden sind, Gerichte anzurufen (so Gerhard). Allgemeiner formuliert: Ist es legitim, geschlechtsspezifische Unterschiede hierarchisch zu interpretieren. Die Antwort ist einfach. Für eine empirische Wissenschaft ist es

1 ZRRSoz 2 $/ 8_{4}$, mit Betragen von Doris Lucke, Die Frauenforschung und ihre juristusche Abnchmer, S. 203 ff.; Ute Gerhard, Warum Rechismeinungen und Unrechtserfahrungen von Fiauen niche zur Sprache kommen, S. 220 ff. (eme Repltk auf Rudiger Lautmann, Negatives Rechtsbewußtsein - Uber Geschlechtsdifferenzierungen in der juristischen Handlungsfahigkeit, ZIRSoz 2/80, S. 165 ).

2 So Lautmann im Vorwort, den in der untersten Stufe der jursstischen Karriere unter $18 \%$ licgenden Frauenantel wohl etwas uberschatzend.

3 Vgl die getrennten Veroffentlichungen: Ute Gerhard, Gleschheit ohne Angleschung, 1990; Rudiger Lautmann, Gleichheit der Geschlechter und die Wirklictikeit des Rechts, 1990.

$4 \mathrm{Vgl}$. die Rezension von Margarecha Sudhof (in diescm Hefe) und (zu Gerhard) von Jutta Limbach, in: Soziologische Revue 1992, 182 . 
schlicht ein Kunstfehler, wenn Antworten, die durch andere Erfahrungen und Interessen geprägte Einstellungen und Verhaltensweisen dokumentieren, als Defizit wahrgenommen werden und die strukturell bedingte Differenz nicht gesehen wird.

Mittlerweile ist der Disput über "skeptisches o oder "negatives " Rechtsbewußtscin gedeutet worden als die Irage nach dem Verhältnis von Gleichbeit und Differenz. Ute Gerhard betont in ihren 1990 veröffentlichten Buch über "Gleichhcit ohne Angleichung « die produktive Spannung beider Prinzipien. Sie hofft - kontrafaktisch - auf eine Kultur, in der grundlegende Differenzen nicht hierarchisch (besser/schlechter), sondern egalitär (andcrs, aber gleichwertig) interpretiert werden. Lautmann hingegen sieht auch 1990 kein Problem darin, Ungleichheit als Folge von Benachteiligung und damit als Defizit zu interpretiercn.

Nun kann man über theoretische Fragen streiten und dennoch den je anderen Standpunkt anerkennen. Aber genau dies geschieht nicht. Fast tragisch mutet die Art und Weise an, in der das Scheitern eincr auf Kooperation angelegten Arbeit verschwiegen und nur in kleinen polemischen Seitenhieben erwähnt, aber nicht verarbeitet wird. Wieso widerlegen sich die beiden Protagonisten nicht in der ansonsten (innerhalb derselben Zunft) üblichen Form. Es gibr schließlich Vorbilder für berühmte Dispute, die Wissenschaftsgeschichtc gemacht haben. Im allgemeinen dient Nicht-Rezeption weder der eigenen Reputation noch der Wahrheitsfindung. Oder geht es um erwas anderes - um die Verteidigung einer disziplinären Machtposition: Rechtssoziologic versus Frauenforschung? Ein fatales Mißverständnis. Zwar sind mir als Universitärsjuristin Strategien des wissenschaftsinternen Machtgewinns geläufig. Aber können es sich eine prominente Vertreterin der feministischen Rechtskritik und ein engagierter Vertreter des kleinen Fachs Rechtssoziologie leisten, die jeweils andere Fragestcllung innerhalb der ohnehin schwach repräsentierten Grundlagenfächern in dieser Form zu ignorieren' oder (so Lautmann 1990, S. 243/244) gar zu ridikülisicren? Die Fragc stellen, heißt sie verneinen.

Vertreter der etablierten dogmatischen Fächer in Juristischen Fakultäten wissen, daß sie sich schlechte Umgangsformen mit den weniger etablierten Orchideenfächern leisten können. Ihre Strategic der Nicht-Thematisierung oder - im günstigen Fall der selektiven Verwendung empirischer Untersuchungen ${ }^{6}$ ist bekannt und erforscht; etwa in dem von Ulrich Beck und Wolfgang Bonß betreuten DFG-Schwerpunktprogramm über die Verwendungszusammenbänge sozialwissenschaftlicher Ergebnisse ${ }^{7}$.

Doris Lucke, wohl unterrichtet über das Brcmer Projekt und zugleich einbezogen in das "Verwendungsprojekt" von Beck/Bonß, verbindet den frauenpolitischen und den wissenssoziologischen Diskurs und baut cinc Brücke, die aus dem disziplinären

5 Die Tendenz des sich Ignorierens deutet sich bereits $198_{4}$ auf einer von Ute Gerhard und Jutta Limbach veranstalteten Tagung zum Bremer Projekt an: Gerhard/Limbach (Hrsg.), Rechtsalltag von Frauen, 1987. Lautmanns Position ist im Tagungsband nicht mehr vertreten. An seiner Stelle diskutiert Blankenburg die Frage: Haben Frauen ein anderes Rechtsbewußtsein als Manner?, ebd. S. 143. Blankenburg nimmt in diesem Beitrag Lautmanns These auf, nummt ihr aber die frauenpolitische Spitze. Alle "Rechtsglaubigen *, micht nur Frauen, zeigten Symptome snegatıven Rechtsbewußtscins $\alpha$. Aber beı gleıcher Konfliktkonstellavon wurden Frauen ebenso haufig klagen we Manner. Der Mangel sei also nicht geschlechtsspezifisch zu erklaren. Mit anderen Worten: Blankenburg bestatigt Lautmanns theoretısche Annahme, er stimmt auch der Eirschatzung zu, daß fethlende Beretschaft zur instrumentellen Rechtsdurchsetzung etn "Defizit $\alpha$ ware, aber er widerlegt ihn empirisch. Ein paradoxes Ergebus.

6 Selektiv meınt: Verwendung nur dann, wenn die Ergebnısse der Nachbardisziplin dic auf andere Weise gewonnene eigene Losung bestatigen. Vgl. hicrzu Doris Lucke, Verwendung soziologischen WIssens in juristischen Zusammenhangen, ZfRSoz 2/1988, S. $12 \mathrm{ff}$.

7 Beck/Bonß (Hrsg.), Weder Sozıaltechnologic noch Aufklarung? Analysen zur Verwendung sozıolog1schen Wissens, Frankfurt 1989 . 
Kleinkrieg herausführt ${ }^{8}$. Ich überspitze ihre Deutung: die unhöflichen Umgangsformen der Juristen mit den Rechtssoziologen sind eine wenig souveräne, aber machtbewußte Antwort auf einen gestiegenen Legitimationsdruck. Sie trifft eine ursprünglich hermeneutische Disziplin, die ohne Rückgriff auf Sozialwissenschaften ihre Aufgabe darin sah, Interessen zu bewerten und Rechtsbegriffe analytisch zu klären. Der Zwang zur Verwissenschaftlichung erfaßte auch die Gesetzgebung und Rechrsanwendung. Selekrive Verwendung oder strategische Nichtverwendung sozialwissenschaftlicher Studien ist offenbar ein - langfristig zum Scheitern verurteilter, abcr kurzfristig effektiver - Versuch, sich gestiegenen Anfordcrungen zu beugen und zuglcich zu cntziehen: cine für die juristische Kunst typische, flexible Reaktion.

Wie verhalten sich nun die juristischen Abnehmer der Frauenforschung? Sie ignorieren sie oder verwenden sie strategisch, das meint Juristen bauen selektiv passende Ergebnisse in das eigene Bcgründungssystem ein, um »sozial wissenschaftlich" abzusichern, was aus normativen Vorgaben gefolgert wurde. Mit anderen Worten: die Ergebnisse der Verwendungsforschung lassen sich mühelos auf dieses Feld übertragen. Es wiederholen sich auch die zwischen Rechtsdogmatik und Rechrssoziologie cingcspielten schlechten Umgangsformen. Neu ist lediglich, daß sich ein vergleichbares Muster nun auch im Umgang der beiden kleinen Fächer feministische Rechtskritik und Rechtssoziologie wiederholt. Allerdings mit einem nicht unbeachtlichen Unterschied: dort machr das Machtspiel keinen Sinn, da es hier kein nennenswertes Gefälle an Reputation gibt. Beide Disziplinen leiden vielmehr - im Verhältnis zur Rechrsdogmatik - darunter, daß ihnen die für eine angemessene Rezeption norwendige interdisziplinäre Anerkennung oft verweigert wird. Wieso spielen sie dann ein Spiel, das für beide ein Verliererspiel sein muß? Rechtssoziologen wie Lautmann - so meine Deutung - haben aus ihrer Situation der fachlichen Überlegenheit in empirischen Fragen, aber strukcurelle Unterlegenheit, was die Umsetzung ihres Wissens betrifft, etwas zu schnell gelernt. Er dreht - wenn die Kollegin Fraucnforscherin ist den Spieß um und antwortet mit Nichr-Thematisierung, Ridikülisierung, Techniken also, die langfristig sein eigenes Anliegen, die Geschlechterfrage empirisch präzise und theoretisch reflektiert zu behandeln, untergraben. Ein Eigentor also. Doris Lucke empfiehlt Gelassenheit. Advokatorische Frauenforschung muß ihre eigene Reputation erhöhen, den Rahmen des geltenden Rechts- und Rechtsanwendungssystems dogmatisch und empirisch studieren und Verwendungszusammenhänge erkennen?.

Nicht nur inhaltlich, auch methodisch gibt es einige wunde Punkte. Während Lautmann versucht, den Umgang von Frauen mit Recht und Rechtsinstanzen nach dem Mobilisierungsansat ${ }^{10}$ zu erklären, die Frauenfrage also als Anwendungsbeispiel für ein ihm geläufiges Phänomen anzusehen, betont Ute Gerhard die Eigenständigkeit der Frauenforschung. Es sei nicht nur schwierig, unter anderen Fragestellungen gewonnenes Material "nachträglich" geschlechrsspezifisch auszuwerten, polemisch ausgedrückt: sich sozusagen kostengünstig auch noch beim Thema "Frauen und Recht « zu profilieren, sondern irreführend bis falsch. Einer »zunächst vernachlässigten Forschungsfrage " eröffnet eine sekundäre Auswertung "möglicherweise auch keine neucn Dimensionen " ". Sie schlägt startdessen vor, die ganzheitliche Kategorie

8 Doris Lucke, wie Fn. 1 ; dies. Das Geschlechtsverhaltnis im rechtspolttischen Diskurs. Gleichstellungsdiskussion und gesetzgeberischer "double talk ", bremer sortologische lexte 1989, insb. S. 13-34.

9 Dies., ZfRSoz $2 / 84,216$; dies., 1989 , S. 56 .

10 Blankenburg, ZfRSoz 1:1980, 33 ff.

I1 So kritisch Gerhard, ZfRSoz $2 / 84$, S. 225 , zur nachworlichen Auswertung der 1982 durchgefuhrten Befragung von Blankenburg/Reifner/George's/Tiemann, Rechtsberatung. Sie fuhrt-leider kommentarlos zur Widerlegung der Position Lautmanns, vgl. Blankenburg, in: Gerhard/Lımbach, wic Fn. 4 , S. 143 . Es 
des "weiblichen Lebenszusammenhangs" und die spezifischen "Unrechtserfabrungen * von Frauen zur Interpretation der in den Intcrvicws geäußerten Frustrationen heranzuziehen. Diese, wie sie meint "andercn ", nämlich qualitativen Forschungsmethoden seien dem Thema: Frauen und Recht angemessener als die der quantitativ verfahrenden »männlichen « Rechtssoziologie, die sich darauf beschränke, zu zählen, wer wann welche Instanzen mit welchem Erfolg mobilisiere.

Ich habe einige Schwierigkeiten mit diesen Zuschreibungen. Wenn sich die Annahme als richtig erweisen solltc - trotz belcgbarer $Z$ weifel -, daß Frauen bei vergleichbaren Ausgangssituationen seltener Gerichte anrufen und als Klägerinnen, Antragstellerinnen oder Beklagte weniger erfolgreich sind, weil sie nicht stratcgisch vorgehen, sondern defensiver agieren, dann ist zunächst zu fragen, ob sie sich wirklich auch in vergleichbaren Konfliktkonstellationen befinden; eine für die Verfahrenssoziologie so grundlegende Kategorie, daß sie nicht vernachlässigt werden darf, sondern - wenn nötig - durch Verwendung der Frauenforschung erhellt werden muß. Es kann dann aber auch nicht ausgeschlossen werden, daß eine "männliche» Rechtssoziologie die Frage des "weiblichen Lebenszusammenhangs « klären muß. Sollte sich aber zeigen, was zu vermuten ist, daß sich die Frauen in den beobachteten Situationen in geschlechtsspezifisch geprägten und damit ungleichen Konstellationen befunden haben, dann handelt es sich aus rechtssoziologischer Perspektive nicht um ein Problem des "männlichen « oder "weiblichen « Rechrsbewußtseins, sondern um ein Problem der ungleichen Mobilisierbarkeit von Recht in sozialen Konflikten (vgl. hierzu Kap. III. Haben Frauen ein anderes Rechtsbewußtsein als Männer?).

Es kann - so meine These - keine unüberbrückbaren Gräben zwischen empirischer Rechtssoziologie und Frauenforschung geben (Vorurteile schon). Aus diesem Grund wähle ich im folgenden (II. Die Glcich heit der Geschlechter und die Wirklichkeit des Rechts) die Technik der immanenten Kritik. Ich versuche zu zeigen, was eine - in meinen Augen von Ute Gerhard zu schnell abgefertigte - sekundäre Auswcrtung von rechtssoziologischen Studien für dic Frauenforschung an Resultaten erbringen kann und wo Grenzen erreicht sind. Ich werde zeigen, daß auch aus der Pcrspektive des von Lautmann vertretenen Mobilisierungsansatzes (Blankenburg) sein Ergebnis unhaltbar ist. Selbstverständlich kann das Thema "Frauen und Recht" in Kooperation mit Kollegen, die an Frauenforschung nur am Rande interessiert sind, betrieben werden. Aber es setzt voraus, daß alle Beteiligten bereit sind, die jeweils andere Fragestellung und Methode kritisch im Kontcxt der eigenen Thematik zu diskutieren und niche so zu verpacken, daß man sie zum Verschwinden bringt. Disziplinäre Machtspiele - auch wenn sie irrational, nämlich als doppeltes Verliererspiel inszeniert werden - verstärken die ohnehin vorhandenen Scheuklappen.

\section{Die Gleichheit der Geschlechter und die Wirklichkeit des Rechts}

Thema der von Lautmann präsentierten Studie ist nicht Gleichheit schlechthin, sondern die spezifische Rolle von Recht bei der Durchsetzung von gleichen Chancen. Die Studie befaßt sich also mit Themen, die zwar geläufig sind, die aber von keiner Disziplin in diesem Zusarmmenhang behandelt werden. Im Gegenteil: sic werden von einer androzentrischen Soziologie (bisher) als feministisch gemieden und sind für eine feministische Debatte (noch) zu juristisch bzw. zu rechtssoziologisch.

Das empirische Material ist Projekten zu folgenden Themen entnommen: "Rechtsbewußtsein*, "Arbeitsvermittlung "und "Ehescheidung". Das Teilprojekt "Rechtsbewußtsein « ist eine Sekundärauswertung der von Ute Gerhard durchgeführten 
Interviews, wobei Lautmann sich nur dafür interessiert, ob Frauen Rechtsansprüchc, die zumindest dem Programm nach einem egalitären Rechtsdenken verpflichtet sind, auch strategisch umsetzen. Kapituliercn sic vor der wstillen Gewalt" (Lautmann 1972) oder verstehen sic es, sie für ihre Zwecke zu mobilisicren'2. Die Inhalte, an denen die strategischen Fähigkeiten überprüft wcrden sollen, sind dem zweiten und dritten Teilprojekt entnommen. Sie behandeln Reformgesetze, deren politischc Zicle als emanzipativ gelten und die es ermöglichen, den weiten Bogen zu den im ersten Teil skizzierten soziologischen Gleichheitstheorien zu spannen. Ausgewählt werden zwci klassische Themen, Familic und Arbeit: das 1976 reformierte Ehescheidungsrecht und das 1969 neuformulierte Arbeitsförderungsgesetz.

Beginnen wir mit dem ersten Teil, den soziologischen Gleichbeitstheorien und den unterschiedlichen Gerechtigkeitserwartungen, die Fraucn und Männer formulieren. Fragt man Frauen und Männern nach den von ihnen bevorzugten Populärideologien zu Gleichheit, Gerechtigkeit etc., dann bevorzugen - erwartungsgemäß - Männer das Vertcilungskriterium Chancengleichheit oder wequity", Frauen die faktische Gleichbehandlung. Geradczu klassisch und absolut rollenkonform orienticren sich die männlichen Bcfragten eher an leistungs- und erfolgsbezogenen Maßstäben. Sie nennen als dazu passendes Verhalten ein eher strategisches Handeln, das auf der Konkurrenz zwischen Gruppen und Personen basiert. Frauen hingegen tendieren in ihren Antworten zu egalitären Konzepten und betonen den Wert intersubjektiver Verständigung und koopcrativer Verhaltensweisen. Soweit dic Selbststilisierungen. Wer glaubt schon, daß Männer tatsächlich mchr »leisten « und Frauen tatsächlich »kommunikativer « sind? Sie sind es jeweils in einem tradierten geschlechtsbezogenen Sinne.

Wie aber erklären sich die stereotypen Antworten? Lautmann besteht darauf, daß empirisch beobachtbare Differenzen zwischen den Geschlechtern nicht mit einem "weiblichen « bzw. "männlichen « Wesen (Natur) erklärt werden können. Sie seien Folge ciner differenten "sozialen Identität «. Dies ist sicher richtig, aber die begründete Abneigung gegen Biologismus soltte nicht zu einem Denkverbot führen. Natürlich gibt es biologische Unterschiede zwischen den Geschlechtern. Das Problem ist, daß herkömmlich aus dicsen Unterschieden unsinnige Schlüsse gezogen wurden.

Ich bin mir nicht sicher, ob die ausschließliche Betonung des "Sozialen « heute noch hilfreich ist. Sie ist notwendig gewesen, solange eine starre patriarchale Denk- und Lebensgewohnheit dazu führte, Differenzen ausscbließlich hierarchisch zu interpretieren; und dies wird auch noch einige Zeit der Fall sein. An Biologie geknüpfte soziale Ungleichhcit ist in meinen Augen die Folge hierarchischer Deutungen, nicht die norwendige Konsequenz der beobachtbaren Geschlechtsdifferenzen. Man kann nun die Biologie für unwichtig ansehen und in beobachtbaren Differenzicn sozialisationsbedingte Unterschiede sehen. Dies ist aber nur ein Fortschritt, wenn man sich zugleich klarmacht, daß auch sozialisationsbedingte Unterschiede hierarchisch gedeutet werden können; aus dem "schwachen « oder "schönen " Geschlecht wird nun ein "wenig erfolgreiches, also in der Konkurrenz unterlegenes Geschlecht $*$, dem man durch Aufklärung und Erzichung auf die Beine helfen muß; oder durch Recht.

warc sehr nutzlich gewesen, wenn die zum Zeitpunke der Erstellung des Manuskrıpts bereits bekannte Kontroverse zumindest klarstellend berucksichtigt worden ware. Zu Blankenburg u. a. 1982, vgl. Frommel, ZRP 1983, 31 (damals unter Vernachlassigung der in der Studie ebenfalls ignorierten Frauen(rage).

12 Wer die Debatte um die Reform des $\$ 2$ I 8 SrGB verfolgt, kann nicht behaupten, daß es welcher Frauenpolitik auch immer gelingen konnte, vor den Toren der Jurisprudenz symbolische Siege zu erringen, was nicht heißt, daß sie sich praktisch den Zumutungen beugt. 
So gesehen macht es Sinn, mir Gerhard die Differenz der Geschlechter und nicht wie Lautmann - „Defizite von Frauen zu beschreiben. Folgt man dicsem Ansatz, dann verändert sich der Blick: angeset $\angle t$ wird bei hierarchischen (d. h. willkürlichen, lediglich auf herkömmlichen Präferenzen beruhenden) gesellschaftlichen und rechtlichen Bewertungen bestimmeer - geschlechtsspezifisch bedeutsamer - Verhaltensweisen und Lebensformen. Wieso solltc, um die Beispiele aus den Populärideologien zur "Gleichheit « auf «unehmen, strategisches = »männliches Handeln »rational» sein, das eher kommunikative $=$ "weibliche " hingegen nicht? Sollen doch androgyne Frauen und Männer differente Stile mischen. In der sozialen Realität tun sie es ja (ansatzweise) auch schon. Mit anderen Worten: ich bin es leid, zu bestreiten, daß es biologische und sozial nicht beliebig manipulierbare Differenzen gibt. Ich finde es einleuchtend, daß das differenziertc kulturelle Wissen von Frauen und Männern bedeutsam ist. Es ist offenbar für dic eigenc Identität wichtig, zeugen oder gebären zu können, auch wenn diese biologische (und kulturelle) "Leistung " von Einæclnen bewußt und unbewußt verweigert wird. Sicher, diese Fähigkeiten werden historisch und sozial überformt, aber übrig bleiben: biologische, sexuellc, kulturclle und soziale Differenzen. Die Betonung des Sozialen mag professionspolitisch befriedigen, aber sie verleitet Lautmann in dicser Studie zu einigen voreiligen Festlegungen.

Die folgenreichste Festlcgung bexicht sich auf dic feministische Kategorie dor "Geschlechtsrollenidentität», so könnte man gender übersetzen. Eine Begriffsklärung fehlt bei Lautmann, weil er die Debatte in seinem Buch nicht erwähnt: ein Phänomen der Nicht-Rezeption. Aber er schweigt nicht, sondern weiß es besser: Auf S. 258 etwa verweist er - affirmativ - auf einen engen soziologischen Rollenbegriff (ohne Diskussion der theoretischen Konsequenzen der beiden unterschiedlichen Diskurse). Eine Rolle im soziologischen Sinne können Männer und Fraucn ausfüllen. Der Rollenbegriff ist entweder sonderbar geschlechtsneutral oder er reduziert »Geschlecht" auf cinige wenige Rollen, nach dem Motto: "weiblich sein heißt "Hausfrau «, "Mutter « und "Ehefrau « oder "Geliebte«, "Karrierefrau « sein. Dic Identität der Rollenträgerin und dic soziokulturellen Regeln der differenten Wahrnehmung von Frauen und Männern bleibr ausgespart. Die "Geschlechtsrolle" ist also keine "Rolle« im klassischen Sinne. Ich will und kann diese Debatte hier nicht vertiefen, aber gezeigt werden soll, daß Nicht-Rezeption folgenreich ist. Am Ende wird die Darstellung konfus, weil Lautmann eine angebliche Distanz »der Frauenforschung* gegenüber "der Soziologie» und deren "Rollenbegriff» kritisiert, eine Distanz, die mir in dieser Kürze nicht nachvollziehbar ist, zumal er auch an dieser Stelle nicht auf die feministische Debatte über Geschlechtsrollen i.S. von sexueller und soziokultureller Identität (gender) eingeht. Stattdessen folgt eine polemische Breitseitc. Gegeneinander ausgespielt wird »die Soziologie " gegen »den Feminismus «. Als »Juristin " könnte mir das doppelt gleichgültig sein, da sich die Vertreter meines Faches beiden Disziplinen gegenüber arrogant verhalten. Aber weil dies so ist, fält mir auf, daß der Autor schon einmal "die Soziologie" gegen "die Rechtswissenschaft» ausgespielt hat. Ich ertappe ihn also bei einer (traumatischen?) Wiedcrholung. Vor zwanzig Jahren gab es professionspolitische Hahnenkämpfe zwischen Juristen und Rechtssoziologen (u. a. Lautmann). Rückblickend erweisen sie sich als irreführend. Auch damals wollte man allen Ernstes "die Rechtsdogmatik « durch "Rechtssoziologie« ersetzen. Mittlerweile kennen wir konservative Soziologen und kritische, sozialwissenschaftliche Fragestellungen gegenüber durchaus aufgeschlossene Juristen. Unterscheiden wir also zwischen einer starren und eincr angemessenen dogmatischen Lösung. Monolithisches Blockdenken ist selten sinnvoll.

Lautmann hätte sich und seinen Lesern viel Mühe erspart und zahlreiche Leserinnen gewonnen, wenn er schlicht darauf hingewiesen hätte, daß das Thema "Geschlechts- 
rollenidentität « eine cigene Diskussion crfordert, dic er an dicser Stelle nicht leisten kann und auch nicht will. "Soziokulturelle Identität «, "Rolle" oder "gender», es sei dahingestellt: jedenfalls gibt es cinc cmpirisch meßbare Differenz in den Gcrechtigkeitserwartungen, verkürzt ein eher weibliches Gerechtigkeitsideal.

Soll dieses juristisch umgesetzt werden, dann bedeutet dies nach Lautmann mehr egalitäres Recht. Ich wäre an dieser Stelle radikaler und würde sagen: eine andere Gerechtigkeitserwartung erfordert eine systematische Überprüfung des rechtstheoretischen Rahmens und der jeweiligen Regelungen. In allen Regelungstypen könnten sich androzentrische Unterstellungen verbergen, doch das Buch, das davon handeln würde, wollte Lautmann nicht schreiben. Ausgewählt hat er das Beispiel des Familienrechts. Was dessen Geschichte bctrifft, verweist er auf Christa Pelikan, die etwa folgende historische Linie zeichnet: Typologisch könne eine Entwicklung von eher

- protektivem Recht (Problem: Ambivalenz von Schutz. und Kontrolle) zu

- kompensatorischem Recht (Problem: schwache gerichtliche Durchsetzung) rekonstruiert werden. In der Gegenwart tendiere dic Gesetzgebung zu

- formal-egalitärem Recht (Problem: Ineffektivität).

Lautmann sieht sehr wohl die Tücken dieser Trias: Das protektive Recht ist zwar im allgemeinen gut durchsetzbar, tendiert aber zur mittelbaren Diskriminicrung (bis hin zur Bevormundung). Genau spiegelbildlich verhält es sich mit den beiden anderen Regelungstypen. Sic sind am Gleichheitsideal orientier, aber wegen ihrer spezifischen Regelungstechnik gerichtlich schwer durchsetzbar. Kompensatorisches Recht wird in Rechtssätzen formuliert, die offen gefaßt sein müssen und daher in der Rechtsdurchsetzung erhebliche Ermessensspielräume aufweisen, die nicht im Sinne der Programmatik genutzt werden müssen. Formal-cgalitärc Normen tendieren darüber hinaus als reine Lippenbekenntnisse dazu, an der sozialen Ungleichheit nichts zu ändern. Wenn cs also tatsächlich eine politische Mehrheit für effektive Gleichheitsrechte gäbe, dann müßte diese material-egalitäres Recht schaffen (S. x 16). Aber dicses würde schon auf der Gesetzgebungsebenc auf massiven Widerstand stoßen.

Damit ergibt sich aus meincr Sicht folgendes Dilemma. Das zur Zeit durchsetzbare Recht mag zwar auf der Programmebene kompensatorisch bis egalitär konzipiert sein, aber es entfaltet paradoxe situative Konsequenzen. Die ambivalenten Wirkungen zeigen sich crst auf den der Normsetzung folgenden Implementationsebenen (Rechtsprechung, Einzelfallentscheidung, Vollstreckung). Letztlich bestimmen also nicht die im Normtext formulierten, sondern die seine Anwendung bestimmenden informellen Regeln die Reichweite eines Reformgesetzes - offenbar ein Strukturmerkmal jeden Rechts, das soziale Gegebenheiten verändern will. Die Rechtsnehmerinnen können sich also nicht auf ihr "Recht « berufen, sondern müssen sich in einen komplizierten und ambivalenten Aushandlungsproze $\beta$ begeben.

Lautmann sieht im Ehescheidungsrecht ein »Musterbeispiel formal-egalitärer Gesetzgebung "(S. I I 7). Dies trifft sicher für die Gesetzessprache zu. Es ist durchgängig von "den Ehegatten “ die Rede. Auch die Regelungstechnik läßt sich so kennzeichnen, da zumindest auf der Oberfläche alle Ansprüche umkehrbar sind. Materiell ist eine formale Gleichbehandlung bei der Regelung des Versorgungsausgleichs, also in cinem außerordentlichen wichtigen Teilbereich, gelungen, das auch in der Praxis insgesamt gut umgesetzt wird.

Darüberhinaus ist cs problcmatisch, ausgerechnct das Eherecht als Beispicl für formal-egalitäres Recht anzusehen, da es im Kontext des Steuer-, Arbeits- und Sozialrechts abgeleitete Unterhalts- und Versorgungsansprüche regelt, also einen Versor- 
gungstypus, der auf der herkömmlichen geschlechtsspezifischen Ungleichhcit beruht. Lautmann weiß dies, denn er macht schließlich eine begriffliche Unterscheidung zwischen formal- und material-egalitär. Aber er erklärt nicht die Konsequenzen, die diese Unterscheidung hat. Weitere Mißverständnisse resultieren daraus, daß er das formalegalitäre Programm in den Vordergrund stellt und darauf verzichtet, auf cinschlägige Studien zu seiner Implementation zu verweisen. Lautmann kannte sie, aber er hat sie aus Gründen, der mir nicht ersichtlich sind, nicht in seinen Text eingearbeitet, und er hat auch keine salvatorische Klausel eingefügt, die die Leser auf diese Begrenzung aufmerksam macht's.

Welche Widersprüche sind zu konstatieren? Auf der Programmebene will das reformierte Recht u. a. ehebedingte Nachteile des die Kinder betrcuenden Ehegatten ausgleichen. Die scheinbar geschlechtsneutrale Sprache kann nicht darüber hinwegtäuschen, daß es so gut wie kcine Väter gibt, die ihren Beruf aufgeben, um kleine Kinder zu betreuen. Kompensatorische Ansprüche werden also in der Praxis ausschließlich von Frauen geltend gemacht. Fragen wir daher, wic diese Normen praktisch umgesetzt werden. Die einschlägigen Studien zeigen übereinstimmend, daß das neue Recht zwar beim Versorgungsausgleich Vorteile gebracht hat, aber im Ergebnis nicht zu einer kompensierenden Umvertcilung zwischen den Ehegatten führt, sondern im wesentlichen der Sicherung des Kindesunterhalts dient. Dies ist zwar ein eminent wichtiges Ziel, aber keines, das der Gleichstellung der Ehegatten dient, sondern dem Kindeswohl't.

Weitere Schritte in Richtung einer schon eher materialen Gleichberechtigung wären, ein oder mehrere Erziehungsjahre staatich zu subventionicren, generell Teilzeitarbeit zu fördern und sog. Familienphasen mit Wiedereingliederungshilfen zu verknüpfen. Schließlich wäre eine aktive Arbeitsmarktpolitik und eine Verbesserung der außerhäuslichen Kinderbetreuung erforderlich. Ansätze gibt es, aber aus meiner Sicht befinden wir uns noch lange nicht in einer Phase konsequenter egalitärer $\mathrm{Fa}$ milien-, Sozial-, Arbeits- und Rechtspolitik. Man kann über die Begriffe streiten, aber auch ein formal-egalitäres Recht müßte folgende Mindestanforderungen erfüllen. Es müßte dic Leistung »des Ehegatten», der Kinder und andere Hilfsbedürftige betreut, also in der Regel die Frau, angemessen bcwerten ("männliches und "weibliches « Gerechtigkeitsideal: equity und Gleichbehandlung). Da diese Arbeiten mit Benachteiligungen auf dem Arbeitsmarkt erkauft werden, müßten sie wenigstens versorgungsrechtlich anerkannt werden, etwa relevant sein für eine eigenständigc, also nicht abgeleitete soziale Sicherung. Dies würde noch lange nicht die materiale Gleichstellung durch Recht ermöglichen, aber dennoch erhebliche Veränderungen im Steuerrecht, Sozialversicherungs- und Rentenrecht mit sich bringen.

\section{Haben Frauen ein anderes Rechtsbewußtsein als Männer?}

Ich komme nun zum dritten Schwerpunkt der Studie, der Kontroverse über das angeblich »negative« Rechtsbewußtscin von Frauen. Margarctha Sudhof hat in ihrer Rezension die Vorgeschichte dargestellt. Eine 198 I vcröffentlichtc erste Auswertung

13 Caesar-Wolf/Eidmann, in: Blankenburg/Vorgt (Hrsg.), Implementation von Gerichtsentscheidungen, Jahrbuch für Rechtssoziologie und Rechtstheorie, Bd. 11, 1987; Doris Lucke, Die Praxis des neuen Scheidungsrechis in der Bundesrepublik, Leviathan 1983, S. 537 ; vgl. dzes./Sahine Berghahn, "Angemessenhert a im Scheidungsrecht, 1983, insb. S. r 37 ff.: Weibliche Lebenslaufe zwischen Familie und Beruf und mánnliche Erwerbskarrieren - eine Gegenüberstellung stypischer* Mainner- und Frauenbıographien.

14 Vgl. etwa Frommel, ZfSexf 3/1991, S. 183 
von 40 Interviews von Utc Gerhard crgab eine auffällige Rechtsskepsis von Frauen. Lautmann kannte Gerhards Position, da er Projektleiter war, und publizierte seine Gegenmeinung schon 1980, also ohne den Projektbericht sciner damaligen Mitarbeiterin abzuwarten oder ihr im Rahmen scincr Veröffentlichung Gelegenheit zur Gegendarstellung zu geben (ZfRSoz 2/80, S. 171). Diese erfolgtc erst 1984 (ZfRSoz 2/84, S. 220).

Der Titel "Negatives Rechtsbewußtsein - über Geschlechtsdifferenzierungen in der juristischen Handlungsfähigkeit « zeigt, daß von einer rechtsskeptischen Einstellung unmittelbar auf eine fehlende strategische Kompentenz geschlossen wird. Dieser nicht nur kühne, sondern falsche Schluß führt zurück zum Ersten Teil des Buches. Dort hatten sich geschlechtsspezifisch unterschiedliche Gerechtigkeitserwartungen ergeben. Diese schlagen sich nun in den Antworten zum Themenkomplex $»$ Rechtsbewußtsein « nieder. Aber "Rechtsbewußtsein « ist nicht identiscl mit juristischer "Handlungsfähigkeit火. Letztere meint die Kompetenz, "eigene Intcressen durch aktives Anmelden von Rechtsansprüchen zu schützen und zu verfolgen $\kappa$. Um zu testen, wie Frauen ihre Interessen verfolgen, hätte Lautmann aber eine ganz andere Studie machen müssen. Aus geschlechtsrollenkonformen Äußerungen über Einstcllungen können wir nicht auf entsprechendes Verhalten und schon gar nicht auf fehlende Kompetenzen schließen, zumindest müßte eine empirisch vorgehende Disziplin auf einige Überraschungen gefaßt scin.

Das wertende Attribut »negativ «, das der Verfasser deskriptiv vcrstanden wissen will (dem Kontinuum "negativ-positiv« soll nach Lautmann keine Bewertung unterstellt werden; 1990, S. 244), meint also eine spezifische Einstellung, nämlich eine von den interviewten Frauen auffällig häufig betonte Rechtsskepsis, ein »demonstratives Ignoramus (1990, S. 2 10), wie Lautmann sarkastisch fcststellt. Der Schluß von der "negativen « Einstellung auf cin cntsprechendes Verhalten ist spekulativ gewonnen, und zwar über folgende Gedankenbrücke: Wer eine Situation als eher nicht rechtlich relevant wahrzunehmen geneigt ist, entwickele - so Lautmann - eine eher geringerc juristische Kompetenz, d. h. die Fähigkeit, juristische Instanzen im eigenen Interesse erfolgreich zu mobilisieren. "Positives « Rechtsbewußtsein ist demnach eine stratcgische Einstellung. Bchauptet und mit Antworten aus den Interviews belegt wird die These, daß männliche Befragte häufiger ein strategisches oder instrumentelles Rechtsclenken dokumentierten als Frauen. Aus »männlich «-»weiblich « wird "positiv «-»negativ« (angeblich ohne Wertung).

Die Differenz zwischen den Geschlechtern, die in den Interviews dcutlich geworden war, wird übersetzt in: strategisches Denken und Handcln = "männlich " und ein Defizit an Strategie $=$ "weiblich «. Nicht cinmal im Alltagsbewußtsein würdc so einseitig gewerter werden, sondern wohlwollender: strategisch $=$ rechtlich $=$ männlich versus kommunikativ $=$ außerrechtlich $=$ weiblich. Lautmann übernimmt die stereotype Sicht - mit polemischen Vorzeichen - und legt die Geschlechter aus der Warte der empirischen Rechtssoziologie auf derartig primitive Stereotype fest. Wir wissen aber mittlerweile, daß Zuschreibungen nicht "die Realität « widergeben.

Gerade der von Lautmann als theoretische Hintergrundannahme verwendete Mobilisierungsansatz's, dem ich - im Gegensatz zu Ute Gerhard - folge, zcigt, daß Rechtsdurchsetzung ein komplizierter Vorgang ist. Ihr Erfolg hängt weniger von der strategischen Kompetenz als vielmehr von einer Reihe von strukturellen Voraussetzungen ab, die ziemlich genau beschrieben werden können. Zum Verständnis des Mobilisierungsansatzes sei vermerkt, daß Blankenburg von Annahmen ausgeht, die

is Blankenburg, ZfRSoz 1/1980, S. 33; ders./Reifner, u. a., Rechtsberatung, 1982. 
mit der geschlechtsspezifischen Mobilisierbarkeit von Recht nichts zu tun haben. Es lassen sich nur sekundär einige Ergebnissc übertragen. Ein eigenständiger Beitrag zur Frauenfrage im Recht kann dies nicht sein. Folgen wir also Lautmanns forschungsleitender Frage und konfrontieren wir sie nachträglich mit Erkenntnissen der Biographieforschung.

Warum wird ein Problem zum Rechtsproblem und warum ein anderes nicht? Welche Rolle spielt die Infrastruktur der zugänglichen Rechtsberatung (Zugangsbarrieren zu Anwälten, Netz beratender Institutionen, Kostenfragen) und welche organisationssoziologischen Erkenntnissc gibt es, um dic Häufigkeit und Verteilung von gerichtlichen Verfahren zu erklären (und ggf. zu becinflussen)? Die ersten Ergebnisse zeigen, daß es in erster Linie die Konfliktkonstellation ist, die die Verrechtlichung eines Konfliktes eher nahe- oder fernliegend erscheinen läßt. Ist cine Rechtsposition einmal thematisiert, dann ist für die nächste Schwelle, die Anrufung eines Gerichts entscheidend, wer die Mobilisierungslast trägt und wie groß die Beweisschwierigkeiten sind, ferner die Einschätzung der Erfolgsaussichten und - nicht zu vergcssen - der Zeitfaktor. Letzteres meint Überlegungen wie: Wer kann sich eher eine lange Verfahrensdauer (ggf. auch hohe Kosten) lcisten und wem schadet sie in erster Linie.

Zum Einfluß der Konfliktkonstellation läßt sich ganz generell sagen, daß anonymc, auf den einmaligen Austausch von (geldwerten) Leistungen ausgerichtete Kontakte sich im Falle einer Störung leicht verrechtlichen lassen. Den Gegenpol bilden über einen langen Zeitraum hinweg einzuklagende Forderungen in persönlich gefärbten Beziehungen. Die sozialen Kosten der Verrechtlichung persönlicher Beziehungen sind höher als die punktueller Geschäftskontakte. Gerichtliche Verfahren betreffen also - wenn überhaupt - nur die Beendigung von engen sozialen Beziehungen. Diese Regel gilt ganz generell, nicht nur für das Ehescheidungsverfahren ${ }^{16}$. Ferner läßt sich die generelle Regel formulieren, daß alle Privatpersonen (mit Ausnahme der nicht gerade großen Gruppe der Prozcßerfahrenen) gerichtliche Verfahren (wenn sie können) vermeiden, d.h. sie gehen insbesondere dann nicht vor ein Gericht, wenn es Alternativen gibe (Institutionen der Vermittlung und Schlichtung).

Im folgenden werden die Indikatoren des Mobilisierungsansatzes bezogen auf das Ehescheidungsverfabren. Wie sieht es mit der Wahrscheinlichkeit einer Verrechtlichung der Auflösung einer Ehe aus? Sie ist sehr hoch: ein nur auf den ersten Blick paradoxes Ergebnis, was damit zusammen hängt, daß cs nach deutschem Recht kcine Alternative zu einem gerichtlichen Scheidungsverfahren gibt. Also kann sich die im Einzelfall (und bei Frauen möglicherweise häufiger) anzutreffene persönlichc Ncigung, Beziehungsprobleme nicht als "Rechtsproblem « zu definieren, nicht auswirken. Wer sich endgültig trennen und ggf. neu verheiraten will, muß zu Gericht. Dort bekommen beide, wie ein Praktikerspruch lautet, nicht "Recht «, sondern ein Urteil. Es gibt aber zahlreiche Wege einer konsensualen Lösung innerhalb des gerichtlichen Verfahrens. Diese werden von der überwiegenden Zahl der Scheidungswilligen bevorzugt und führen dazu, daß sie die Familiengerichte lediglich quasi-notariell beurkunden und für vollstrcckbar crklären lassen, was sie auf außergerichtliche Weise ausgehandelt haben. Mit anderen Worten: würde das reformierte Ehescheidungsrecht die Scheidungswilligen nicht in cin förmliches Verfahren zwingen, inncrhalb dessen alle wesentlichen Folgen einer Scheidung zu regeln sind (Verbundsystem), dann wären soziale Zermürbungstaktiken noch wahrscheinlicher als sie

16 Blankenburg u a. haben dies anhand der Befragung von potentiellen Klägern und Klagerınen und der Analyse der Erfolgsaussichten von Klagen gezeigt. Es ist die Quintessenz des sog. Mobılisierungsansatzes, dem Lautmann - im Prinzip - folgt. 
ohnehin sind. Wenn sie vorkommen, dann zielen sie auf den Verzicht von Rechtspositionen, setzen also auf die belastenden Faktoren, die der Mobilisierungsansatz als Gegenindikatoren einer Verrechtlichung beschreibt (etwa den Zeitfaktor). Das von Blankenburg in eine Theorie gefaßte Erfahrungswissen wird strategisch eingesetzt. Strategisches Geschick sctzt allerdings voraus, daß man sich in einer strategisch günstigen Position befindet. Diese scheinen ungleich verteilt zu sein.

Schon ein erster Blick auf die von Blankenburg beschriebenen Indikatoren erfolgreicher Rechtsdurchsetzung zeigt, daß sie in hohem Maße geschlechtsspezifisch gefärbt sind. Sie haben - so viel läßt sich jetzt schon gegen Lautmann einwenden - wenig mit Eigenschaften, Einstellungen und Verhaltensweisen zu tun, führen also aus der ohnehin fragwürdigen Debatte über typisch "männliche und »weibliche "Eigenheiten hinaus. Entscheidend für erfolgreichc Pokerspiele vor Gericht ist die Art der Forderung, die geltend gemacht oder abgewehrt werden soll, und der Zugang zu professioneller Rechtsberatung. Die Art der Forderungen, die eher von Frauen geltend gemacht werden, sind eher schwierig durchzusetzen, hingegen ist der Zugang zu spezialisierter Reclutsberatung eher gut - dank des Prozeßkostenhilfegesetzes und dcs engen Netzes an spezialisierten Scheidungsanwältinnen.

Ein Beispiclsfall:

Nehmen wir den häufigen Fall einer scheidungswilligen Frau, die das Sorgerecht behalten und Unterhalt für sich (und die Kinder) verlangen will. Gehen wir nicht vom Auslaufmodell der reinen Hausfrauenehe aus, sondern von dem mittlerweile "modernen « der halbtags außerhäuslich erwerbstätigen Mutter mit kleinen Kindern. Sie benötigt einen Unterhaltsanspruch zur Aufstockung des eigenen Einkommens. Im Prinzip sieht das geltcnde Recht dies vor. Aber unter prozeßstrategischen Gesichtspunkten ist sie in der relativ ungünstigen Situation, daß sie die Mobilisierungslast trägt; d. h. sie will etwas, und zwar möglichst schnell. Wer nur eine Forderung abwehren will, hat eine strategisch günstigere Ausgangslage, da die Zeit für ihn arbeitet und er sich deswegen auch ein langes Verfahren leisten kann. Im Ergebnis nützt dieser Vortcil aber wenig, wenn die Forderung, gegen die man sich wendet, rechtlich klar und wenig beweisschwierig ist. Dies ist etwa der Fall beim Kindesunterhalt. Dort ist es leicht, einen Titel zu erringen. Die Probleme beginnen, wenn er vollstreckt werden muß. Die Mobilisierungslast bezieht sich beim Kindesunterhalt also auf die Vollstreckbarkeit, nicht die Rechtsdurchsetzung. Die Zwangsvollstrekkung aber kann insbesondere im Falle eines unversöhnlichen Rechtsstreits unüberwindliche Probleme berciten.

Kehren wir zurück zum Aufstockungsunterhalt. Die Rechtslage ist relativ unübersichtlich, nicht zuletzt wegen des unbestimmten Rechtsbegriffs der zumutbaren "angemessenen * Erwerbstätigkeit (neben der Kinderversorgung). Fragen wir daher nach den übrigen Ansprüchen, die sich gegenüber stehen. Wie sieht es mit vermögensrechtlichen Forderungen aus? Sie sind rechtlich ziemlich leicht zu begründen, aber sie können große Beweisprobleme machen. Sollte etwa eine Antragstellerin nicht gut informiert sein über die Einkommens- und Vermögenslage des zahlungspflichtigen Ehegatten (wichtig für den Zugewinnausgleich), was bei Selbstständigen relativ häufig der Fall ist, dann entsteht im Verbund aller Ansprüche und Gegcnansprüche eine insgesamt wenig übersichtliche Verfahrenssituation.

Exkurs: Gemeinsames Sorgerecht und Mobilisierungslast. Der Vollständigkeit füge ich binzu, daß die neuerdings häufig diskutierte Frage des gemeinsamen Sorgerechts nicht zuletzt auch prozeßtaktisch das herkömmliche Gefüge des Scheidungsfolgenrechts verändert. Es kommt mir also an dieser Stelle nicht auf die materielle Frage an, ob ein gemeinsam ausgeübtes Sorgerecht für die Kinder eine adäquate Lösung ist oder nicht: Aus der Perspektive der Antragstellerin unseres Fallbeispiels bedeutet ein 
Streit um das Sorgerecht eine weitere unklare Rechtsposition. Für alle Mütter, die die tatsächliche Sorge während der Ehe getragen haben, bedeutet die Tatsache, daß neuerdings auch das Sorgerecht zunehmend strittig wird, eine Verschlechterung ihrer prozeßstrategischen Ausgangslage. Man nimmt Müttcrn, die (was die Regel ist) die Kleinkinder versorgt haben, einen bislang garantierten geschlechtsspezifischen Anspruch weg und gibt ihnen keinen Ersatz für die verlorene Erwartungssicherheit.

Angesichts der geschilderten, nicht gerade untypischen Prozeßsituation liegt es für die Antragstellcrin nahe, möglichst schnell einen Vergleich herbeizuführen, der ihr einen Neuanfang (mit allen Vor- und Nachteilen) ermöglicht. Allein aus Gesichtspunkten der Soziologie des Verfahrens wird es nachvollziehbar, wieso sich das Ehescheidungsrecht in der Praxis nicht eignet zur Kompensation sog. "ehebedingter « Nachteile, sprich: zur Bereinigung der Folgen einer traditionalen Arbeitsteilung. Leider gibt es wenig Studien, die Rechtssoziologie, Familienrecht und Frauenforschung verknüpfen. Aber eines wird schon jetzt deutlich: strategische Rechtsdurchsetzung ist für denjenigen leichter, der lediglich Zahlungsforderungen abwehren will. Diejenige Person, die in der Ehe die "weibliche" Rolle gelebt hat, kann sich im Scheidungsfall zwar auf ihr Recht berufen, aber die Durchsetzungsprobleme legen cs ihr nahe, nicht nur auf Rechtsdurchsetzung, sondern zumindest auch auf Verhandeln zu setzen. Damit sind wir bei einer Konstante, die für viele Verfahren, nicht nur das Ehcscheidungsverfahren, bedeutsam ist. Dort wird sie nur besonders deutlich: Rechtsdurchsctzung hat zwei Seiten, eine strategische und eine kommunikative. Welcher der bciden Wege zu einem Urteil oder einem Vergleich oder einer Mischung von beiden angemessener ist, hängt von einer Reihe von strukturell vorgegebenen Faktoren ab: der Konfliktkonstellation und dem Vorhandensein eher seltener Ressourcen, etwa Routine im Umgang mit Rechtsdurchsetzungsproblemen.

Zur Erläuterung wählen wir ein cher fernliegendes Beispiel: eine Geschäftsfrau, die in der angenehmen Situation ist, in der eigenen Firma über eine eigene Rechtsabteilung zu verfügen oder zumindest über routinierte Anwältinnenkontakte, kann sich ein instrumentelles Rechtsbewußtsein leisten - und wird dies tun, selbst wenn sie ganz extrem weiblich sozialisiert worden ist. Klug verhandeln - wird ihre Anwältin sagen - kann sie auch noch innerhalb eines gerichtlichen Verfahrens. Wer sich aber nicht in dieser angenehmen Situation befindet oder schwer beweisbare und damit gerichtlich schlecht durchsetzbare Forderungen geltend machen will, wird in erster Linie streitige Gerichtsverfahren zu vermeiden suchen und erst einmal auf Verhandelo und dann erst auf Klagen setzen.

Zwischenergebnis

Rechtsdurchsetzung hat zwei Seiten, Entscheiden und Vergleichen. Dies heißt in den Chiffren des "männlichen oder "weiblichen* Rechtsbewußtseins, es werden beide oder besser: androgyne Kompetenzen verlangt. Die stereotype Angabe zur Einstellung besagt nichts oder nur wenig über das Verhalten.

Fragen wir weiter. Welche Kompetenzen werden in den Rechtsgebieten verlangt, dic Lautmann untersucht hat. Typischerweise werden von allen Beteiligten im Familienrecht konsensuale Lösungen bevorzugt: von beiden Parteien und dem Gericht wird ein Vergleich angestrebt. Streitige Ergebnisse sind nämlich kontraproduktiv, weil sich dann die Probleme der Rechtsdurchsetzung bei der Vollstreckung wiederholen. Machen wir die Gegenprobe und fragen nach dem Verhalten von Frauen und Männern in Rechtsgebieten, in denen eher strategisches Handeln wichtig ist, etwa im Arbeits- und Mietrecht. Im allgemcinen kommt es hier auf die geschlechtsspezifisch differenten Rahmenbedingungen nicht (direkt, sondern nur über den Umweg bran- 
chentypischer Besonderheiten) an. Klagen Frauen als Arbeitgeberinnen/nehmerinnen oder Vermieterinnen/Mieterinnen, verhalten sie sich ebenso wic Männcr. Es gibr - allen Vermutungen zum Trotz - keine geschlechtsspezifischen Unterschiede im Verhalten'?.

In familienrechclichen Verfahren fehlt es an der Umkebrbarkeit der Positionen. Für Männer war der Heiratsmarkt kein Feld, das ihnen zur Sicherung ihrer ökonomischen Existenz offen steht. Vielleicht ändert sich das, aber noch wissen wir nicht, wclches Rechtsbewußtsein sic entwickeln würden, wenn sie die gesellschaftlichen Rahmenbedingungen hätten, mit denen umzugehen viele Frauen genötigt sind. Dies bedeuter, daß Lautmanns handlungstheoretischer Ansatz zu kurz greift. Noch sind die systemischen Rahmenbedingungen für viele Frauen übcrdeterminiert. Sie können (als Kollektiv) nicht einfach durch "positives Rechtsdenken ihre Situation verbessern. Die Ehescheidung, um ein Beispiel herauszugreifen, erwcist sich für vicle als "Kristallisationskern geschlechtsspezifischer Ungleichheit ${ }^{18}$.

Im folgenden möchte ich erklären, was mit überdeterminierten systemischen Rahmenbedingungen gemeint ist. Sie zeigen sich sehr anschaulich bei einem Vergleich der Alterssicherung von Männern und Frauen. Ausgewählt werden in der hier herangezogenen Studie die Jahrgänge der 1919-1921 Geborenen, also die heutigen Rentner und Rentnerinnen. Kein Mann hat einen abgeleiteten Versorgungsanspruch, aber $80 \%$ der Frauen. Die Renten dieser Frauen sind erheblich niedriger, da die Witwenrente nur $60 \%$ beträgt. Aber auch die Rente derjenigen Frauen, die eine eigene Altersversorgung haben, ist erheblich nicdriger, weil sie im Durchschnitt erheblich weniger verdient haben und Kindererziehungszeiten als Lücken in ihrer Erwerbsbiographie erscheinen'?.

Selbst wenn sich die Verhältnisse im nächsten Jahrtausend bessern sollten, bedeutet dies dennoch, daß nach geltendem Recht Kindererziehung rentenrechtlich als "Nichtleistung "definiert wird, ein krasser Verstoß gegen das "männliche « equityPrinzip. Zugleich aber beruhen alle Renten auf dem Generationenvertrag. Sie werden also von den jeweiligen Kindern der künftigen RentnerInnen finanziert. Das Rentenrecht ist somit ein klassisches Beispiel cines direkt und indirekt diskriminierenden Rechts (zur direkten und deswegen verfassungswidrigen Diskriminierung vgl. den entsprechenden Spruch des BVerfG v. 7. 7. 1992). Interessanterwcise komme es gar nicht darauf an, ob man dieses Recht nach "männlichen " oder "weiblichen" Gerechtigkeitsvorstellungen beurteilt. Es ist nach beiden Idealvorstellungen ungerecht, weil Kindercrziehung der wesentliche input für den output Rente ist und weil es weibliche und männliche Erwerbsbiographicn ungleich behandclt, es honoriert die männliche und bestraft die weibliche, obgleich diese - wegen der Doppelbelastung - rentenrechtlich eigentlich besser (nicht nur gleich) behandelt werden müßte.

Die Ungleichheit der Geschlechter beruht also insofern auf Macht und nicht auf "männlichen" Gerechtigkeitsvorstellungen. Unter dem Strich lassen sich also die "Unrechtserfahrungen« von Frauen (Gerhard) nicht einfach wegerklären. Etikettiert man sie als "Defizit", wie es Lautmann mit der Benennung "negatives Rechtsbewußtsein« tut, dann ignoriert man den sozialen und rechtlichen Rahmen, der die Antworten erst plausibcl macht. Im Ansatz erweisen sich die von Frauen gegebenen

17 Blankenburg wie F.n. s. Was ımmer man gegen die Darstellungsweise einwenden mag, sic provozsert durch abwertende Termins wie etwa sRechtsgläubigkeıt«, um die unrealistıschen Gerechngkeitserwartungen *Prozeßunerfahrener* zu kennzeichnen. Das Ergebnis scheint mir zwingend zu sein.

18 So Lucke, in: Soziale Welt Sonderband 7, 1990, S. 363.

19 Allmendinger/Bruckncr/Bruckner. Ehebande und Altersrente, Soziale Welt 1/1992, S. 90 . Neuerdings: Mechthild Veil, Die Armut ist weiblich. Durch das Rentensystem in die Altersarmut, 1992. 
Antworten nicht als "negativ«, sondern als realistisch. Frauen, so mein Ergebnis, planen ihr Leben auf einem anderen sozialen und rechrlichen Hintergrund als Männer, und zwar alle Frauen.

Sie gehen aber auch unterschiedliche Risiken ein. Die Wahl eines eher konservativen Lebensentwurfs etwa hat ökonomische und rechtliche Konsequenzen. Aber auch der emanzipative Weg ist nicht ohne erhebliche soziale Kosten zu haben. Es sind dies Kosten, die Männer nicht tragen müssen, weil sie (noch) mit einer ziemlich hohen Wahrscheinlichkeit eine Partnerin finden, die sich rollenkonform verhält. Frauen hingegen können realistischerweise nicht damit rechnen, daß "er" seine Rolle tauscht. Sie müssen also neue Wege gehen, wenn sie nicht in herkömmliche Fallen geraten wollen. Sowohl das soziale als auch das Rechts-bewußtsein wird von diesen unterschiedlichen Rahmenbedingungen geprägt. Ebenso das Rechtsverhalten, wenn es sich um Konflikte handelt, in denen nicht umkehrbare Positionen zur Debatte stehen. Der Grund ist strukturell: Rechtshandeln orientiert sich an antizipierten Erfolgs- bzw. Mißerfolgsaussichten. Jede Rechtsdurchsetzung setzt strategische und kooperative Kompetenzen voraus. Statt sie in negativ - positiv einzuteilen, gilt es, sie situativ klug zu kombinieren.

\section{Ergebnis}

Die Rekonstruktion der disziplinären Mißverständnisse hat einiges erbracht. Widerlegt ist die These von der fehlenden strategischen Kompetenz von Frauen. Sie folgt weder aus dem Mobilisierungsansatz noch lassen sich die von den interviewten Frauen geäußerten Enttäuschungen derartig vorurtcilshaft interpretiercn. Es läßt sich viclmehr zeigen, daß die Mobilisierbarkeit von Recht abhängig ist vom Bereich und der Konstellation des zugrunde liegenden Konfliktes. Nicht die Einstellung zum Recht, sondern die Art der Konflikte sind geschlechtsspezifisch geprägt. Frauen machen signifikant häufiger die Erfahrung, daß ihre Interessen schwer zu verrechtlichen sind. Und selbst dort, wo es geschehen ist, ist die Rechtsdurchsetzung nicht einfach. Der Vorsorgeausgleich ist ein Gegenbeispiel. Er ist das Paradebeispiel für einen leicht durchsetzbaren Anspruch, der typischerweise Frauen zugute kommt. Beim Kindesunterhalt gilt ähnliches, aber hier ist es schon komplizierter. Zwar ist die Mobilisierungslast der Rechtsdurchsetzung teicht zu tragen, aber bci der Vollstrekkung wird es oft schwer. Hingegen sind die der Kompensation mehebedingter Nachteile« dienenden Ansprüche klassische Beispiele für Rechtspositionen, über die besser verhandelt als gestritten wird.

Also selbst wenn von allen Inhalten abgesehen und Gerechtigkeits- oder Angemessenheirsfragen ausgeklammert werden - nach dem Motto: „Hier bekommen sie nicht Recht, sondern ein Urteil -, läßt sich das, was Ute Gerhard "Unrechtserfahrung" nennt, verifizieren, nämlich in der neutralen Gestalt erhöhter Mobilisierungslasten. Wer einen Schritt weiter geht und rechtskritisch postuliert: Wenn Recht nur auf Macht oder nur auf Strategie gegründet ist, dann ist es Unrecht, fordert eine spezifisch auf diese Fragen zugeschnittene Frauenforschung im Recht. Einc Entschcidung zu treffen zwischen der einen oder der anderen Perspektive wäre unsinnig. Dennoch scheint es cin starkes Bedürfnis zu geben, das Nebeneinander unterschiedlicher Einstellungen und Interessen destruktiv zu verarbeiten, die andere Position abzuwerten oder zu ignorieren, statt "Gleichheit in der Differenz" zu praktizieren. Die Folgen sind fatal: beide ziehen sich in Spezialgebiete zurück und kämpfen dort wissenschaftspolitisch um Nischen. 
Lautmanns Buch hätte spannend werden können. So ist es zu eincr mühsamen Lektüre geworden, weil es stellenweise polemisch bis falsch und oft schwer verständlich ist. Der Grund: die Brücken zur Frauenforschung fehlen. Aber auch im Ghetto der Frauenforschung droht Nicht-Rezeption, und zwar in beiden Richtungen: wenn dic Fragestellungen, Methoden und Ergebnisse nur dann zur Kenntnis genommen werden, wenn sic ins herkömmliche Schema passen, bleibt Frauenforscherinnen gar nichts anderes übrig, als sich in ihre Spezialgebiete zurückzuziehen. Die Brücken zur herkömmlichen akademischen Lehre und Forschung müssen von beiden Seiten gebaut werden, wenn wir den soziokulturellen Einfluß des Geschlechts (gender) auf die Konstitution des je fachspezifischen Wissens erkennen wollen. Es gibt also langfristig keine Alternative zu Projekten wie dem, dessen Scheitern hier exemplarisch analysiert worden ist. 